A local patient focus group identified the need for trustworthy information, written and reviewed by their rheumatology team to aid self-management. They felt information should be readily accessible, in one place, to re-read / re-listen to, as required.

Objectives: To develop a Rheumatology App, for any patient attending our department, to support self-management.

Methods: We worked with a number of rheumatology patients, of differing ages, the Rheumatology multi-disciplinary team (MDT) and an industry partner, hci. digital, to develop our App, called 'Rheumatology Connect'.

We developed over 30 short videos, provided links to other valuable patient websites e.g. Versus Arthritis, NRAS, NASS, and wrote short articles, to provide basic headline information about various different diseases, common treatments e.g. Methotrexate. We provided easily accessible contact details and information about our clinic locations (including postcodes, bus links, car parking). Video content was written by the clinical authors and filmed by hci.digital.

Patient involvement in designing the layout was particularly important for the App to be easily navigable by all.

We launched the App, in June 2018, at a local patient educational conference. We encourage all patients on regular follow up to download it /use it and remind everyone about it in our clinic letters.

Results: Downloads have been ongoing since launch, with over 1953 downloads and 21930 page views, with peaks around the time of our monthly education sessions when we advertise the App to new patients.

Over $54 \%$ spent between 2 to $5+$ minutes in the App at a time. Most of the videos in the App are about 2 minutes long. Common hits include: videos on medications, various conditions, contact details, clinic locations, and services available. We have had a number of spontaneous contacts from patients saying how valuable they find the information we have given them.

The App aids efficiency by allowing direct phone calls to the correct member of the MDT e.g. rheumatology physio, rather than going through the secretaries. We are also encouraging direct email contact via the App. These aspects are helping us make our services more efficient.

Conclusion: The App continues to be downloaded and used by our patients. It has become an important part of our patient education efforts, especially for those with a new diagnosis.

We have had a significant increase in email contact from patients, allowing us to be more efficient by responding more quickly to patient queries, avoid additional telephone calls and avoid ansa-phone messages.

To date, the App continues to be downloaded by patients and we have received very positive feedback. Having surveyed the users, our next step is to develop interactive PROMS, a calendar reminder function and medicines management function as these have been identified as additions users would find helpful to manage their disease. We have raised funds to develop the App. The updated app should be available by May 2020

Download free from App store (search 'Rheumatology Connect') or go to www. rheumatologyconnect.info.

Disclosure of Interests: None declared

DOI: 10.1136/annrheumdis-2020-eular.5635

\section{AB1348-HPR TELEMEDICINE IN RHEUMATOLOGY: ACCEPTANCE BY PATIENTS AND MEDICAL PRACTITIONERS IN GERMANY (TELERHEUMABB) - INTERIM ANALYSIS}

F. Muehlensiepen $^{1,2,3}$, W. Marquardt ${ }^{2}$, J. Knitza $^{4}$, A. Hueber ${ }^{5}$, M. Welcker ${ }^{6,7}$. ${ }^{1}$ Medizinische Hochschule Brandenburg Theodor Fontane, Zentrum für Versorgungsforschung, Neuruppin, Germany; ${ }^{2} \mathrm{KV}$ Consult- und Managementgesellschaft $\mathrm{mbH}$, Potsdam, Germany; ${ }^{3}$ Witten/Herdecke University, Witten, Germany; ${ }^{4}$ Friedrich-Alexander University of ErlangenNürnberg, University Hospital Erlangen, Department of Internal Medicine 3 - Rheumatology and Immunology, Erlangen, Germany; ${ }^{5}$ Sozialstiftung Bamberg, Sektion Rheumatologie, Bamberg, Germany; ${ }^{6} \mathrm{MVZ}$ for RHEUMATOLOGY Dr. Martin Welcker GmbH, Planegg, Germany; ${ }^{7}$ RheumaDatenRhePort (rhadar), Planegg, Germany

Background: The number of rheumatologists in Germany does not cover the minimum medical need [1]. This implies long journeys and waiting periods for patients in the context of rheumatologists' consultations. International studies suggest that telemedicine could resolve these issues and support health care professionals in rheumatologic care $[2,3]$. So far, comprehensive sustainable approaches are still pending in Germany. By addressing the potential users, TeleRheumaBB investigates whether and how telemedicine can support rheumatologic care in Germany. The study is realized from Oct. 2017 until Jun. 2020. Objectives: The objectives of the study are to evaluate the acceptance and willingness of patients and medical practitioners (MP) to implement telemedicine into rheumatological care as well as to understand how telemedical approaches should be designed to be applied in daily practice.

Methods: The study design contains a mixed-methods approach, which combines qualitative and quantitative data. In the qualitative part of the study 28 in-depth interviews and 3 focus groups involving patients, rheumatologists, general practitioners and stakeholders were conducted. The quantitative part of the study comprised a questionnaire survey with rheumatologists $(n=48)$ and referrers $(n=444)$ and a second questionnaire survey with patients $(n=734)$.

Results:

Qualitative Data: The term "telemedicine" is filled with different meanings. Motives to implement telemedicine into daily practice are patient guidance, information, substantial time savings, desire of a modern practice etc. Reported risks of telemedicine are adverse impacts on the doctor-patient-relationship as well as low benefit with high effort.

Quantitative Data: $62 \%$ of the MPs' surveyed answered that they would like to use telemedical approaches. $81 \%$ of the participants supported doctor-doctor communication via telemedicine. Only $47 \%$ of the MPs' surveyed supported doctor-patients communication via telemedicine. MPs' state that there are barriers which prevent them from implementing telemedicine (89\%). These are among others acquisition of technology, administrative expenses and poor remuneration. $53 \%$ of the patients surveyed stated that they had heard the term telemedicine before. $30 \%$ of the participants answered that they would like to try out telemedicine. 38\% answered with "no" and 29\% with "do not know". Further results from the patient survey will be available in June 2020.

Conclusion: The study results show high acceptance rates of telemedicine regarding doctor-doctor communication. Doctor-patient communication via telemedicine is less accepted. However, MPs' are reporting obstacles preventing the implementation of telemedicine in rheumatology. In order to implement telemedicine in rheumatological care comprehensively, adequate conditions must be established in the German health care system.

\section{References:}

[1] Zink A, Braun J, Gromnica-Ihle E, Krause D, Lakomek HJ, Mau W, et al. Memorandum der Deutschen Gesellschaft für Rheumatologie zur Versorgungsqualität in der Rheumatologie - Update 2016. Zeitschrift für Rheumatologie. 2017;76(3):195-207.

[2] McDougall J, Ferruci E, Glover J, Fraenkel L (2017) Telerheumatologiy: A Systematic Review. Arthritis Care Res (Hoboken); 69(10):1546-1557.

[3] Piga M, Cangemi I, Mathieu A, Cauli A (2017) Telemedicine for patients with rheumatic diseases: Systematic review and proposal for research agenda Semin Arthritis Rheum 47(1):121-128.

Disclosure of Interests: Felix Muehlensiepen Grant/research support from: Novartis, Wenke Marquardt Grant/research support from: Novartis, Johannes Knitza Grant/research support from: Research Grant: Novartis, Axel Hueber Grant/research support from: Novartis, Lilly, Pfizer, EIT Health, EU-IMI, DFG, Universität Erlangen (EFI), Consultant of: Abbvie, BMS, Celgene, Gilead, GSK Lilly, Novartis, Speakers bureau: GSK, Lilly, Novartis, Martin Welcker Grant/ research support from: Abbvie, Novartis, UCB, Hexal, BMS, Lilly, Roche, Celgene, Sanofi, Consultant of: Abbvie, Actelion, Aescu, Amgen, Celgene, Hexal Janssen, Medac, Novartis, Pfizer, Sanofi, UCB, Speakers bureau: Abbvie, Aescu, Amgen, Biogen, Berlin Chemie, Celgene, GSK, Hexal, Mylan, Novartis, Pfizer UCB

DOI: 10.1136/annrheumdis-2020-eular.1865

\section{AB1349-HPR IMPLEMENTATION OF A GROUP EDUCATION SERVICE FOR RHEUMATOLOGY OUTPATIENTS COMMENCING BIOLOGIC THERAPY AT UNIVERSITY COLLEGE LONDON HOSPITAL (UCLH)}

M. Naja ${ }^{1}$, E. Hawkins ${ }^{1}$, S. Moore ${ }^{1}$, M. Castelino ${ }^{1} .{ }^{1}$ University College London Hospital, Rheumatology, London, United Kingdom

Background: Biologic therapies are commonly prescribed in a range of rheumatic conditions. From 2016 - 2018, a mean of 226 rheumatology outpatients at UCLH commenced biologic therapy per year for inflammatory arthritis alone ${ }^{1}$. Patient education is required prior to initiation. At UCLH this is delivered via individual appointments with specialist nurses, contributing to waiting times for nurse appointments and thus delays in starting treatment.

Objectives:

1.To evaluate patient satisfaction following group education.

2.To reduce the burden on specialist nurses delivering multiple individual sessions.

Methods: A once-weekly group education service was set up at UCLH. This comprised: an oral PowerPoint presentation; injection demonstration; question and answer session; and written leaflets. Patients were provided with pre- and post-session questionnaires to evaluate the service.

Results: Over the first five weeks of the service, 18 patients attended group edu cation (a median of 4 patients per session). Nine patients $(50 \%)$ had previously researched the medication via internet search engines. Patients' questions prior to the session were most commonly centred on side effects and long-term risks. All of the patients (100\%) felt that "most or all" of their questions were answered during the session. Patients gave their awareness of the reason for therapy a median score of $7 / 10$ pre-education; this rose to a median of $10 / 10$ post-education. The median score given for confidence to self-administer the drug was 